\title{
Xantogranuloma juvenil de pene
}

\author{
San Miguel Fraile P, Quintana de la Rosa JL, Antón Badiola I, Ortiz Rey JA.
}

Servicio de Urología. Hospital Povisa.Vigo

Actas Urol Esp. 2008;32(5):659-661

\section{RESUMEN}

XANTOGRANUMLOMA JUVENIL DE PENE

Presentamos un caso de xantogranuloma juvenil de pene en un paciente de 30 años de edad que clínicamente fue diagnosticado de quiste de inclusión epidérmica. Describimos la histología y etiopatogenia de la lesión haciendo hincapié en el diagnóstico diferencial con otras lesiones similares con pronóstico adverso.

Palabras clave: Xantogranuloma juvenil. Pene. Inmunohistoquímica.

\section{ABSTRACT}

\section{JUVENILE XANTOGRANULOMA OF THE PENIS}

We report a case of juvenile xanthogranuloma of the penis in a 30 year old patient with clinical suspicion of epidermoid cyst. Histology and ethiopathogenesis are reviewed, with special emphasis on the differential diagnosis with other similar lesions with worst prognosis.

Keywords: Juvenile xanthogranuloma. Penis. Inmunohistochemistry.

$\mathrm{E}$ 1 xantogranuloma juvenil (XGJ) es una lesión benigna infrecuente que clínicamente se caracteriza por la aparición de una o múltiples lesiones papulo-nodulares de color amarillo-rojo en la piel, preferentemente de cabeza, cuello y tórax y que sólo ocasionalmente tiene localización extracutánea. Típicamente afecta a recién nacidos o niños de corta edad, pero aproximadamente entre el 10-30\% de los XGJ aparecen en adultos y generalmente se manifiestan como nódulos cutáneos únicos ${ }^{1}$. La afectación del pene es excepcional y tan sólo hay dos casos descritos en esta localización, según la literatura revisada ${ }^{2}$.

\section{CASO CLÍnICo}

Varón de 30 años de edad sin antecedentes familiares ni personales de interés, que acude al servicio de Urología por presentar una lesión indolora en el surco balano-prepucial de un año y medio de evolución. Clínicamente el paciente presenta sangrado ocasional por traumatismo durante las relaciones sexuales, motivo por el cual acude a consulta de urología.
La exploración física evidencia una lesión ligeramente exofítica de límites bien definidos y coloración amarillenta, que mide $0,7 \mathrm{~cm}$ de diámetro. La analítica de sangre muestra unos niveles de glucosa, colesterol, triglicéridos y lipoproteínas en el rango de la normalidad. Con la sospecha clínica de quiste de inclusión epidérmica, se realiza con anestesia local exéresis quirúrgica de la misma, sin dificultad.

\section{Anatomía Patológica}

Macroscópicamente, fragmento irregular de piel que mide $0,7 \times 0,4 \times 0,3 \mathrm{~cm}$. Al corte se observa una lesión nodular, ligeramente indurada de coloración amarillenta, que contacta con borde quirúrgico de resección.

Histológicamente se observa en dermis superficial y profunda una proliferación de células poligonales de aspecto xantomatoso que muestran citoplasmas amplios, claros y núcleos de pequeño tamaño sin atípia ni actividad mitótica destacable. Entre la celularidad anteriormente descrita se observa un ligero infiltrado inflamatorio linfocitario y frecuentes eosinófilos (Figs. 1 y 2). 


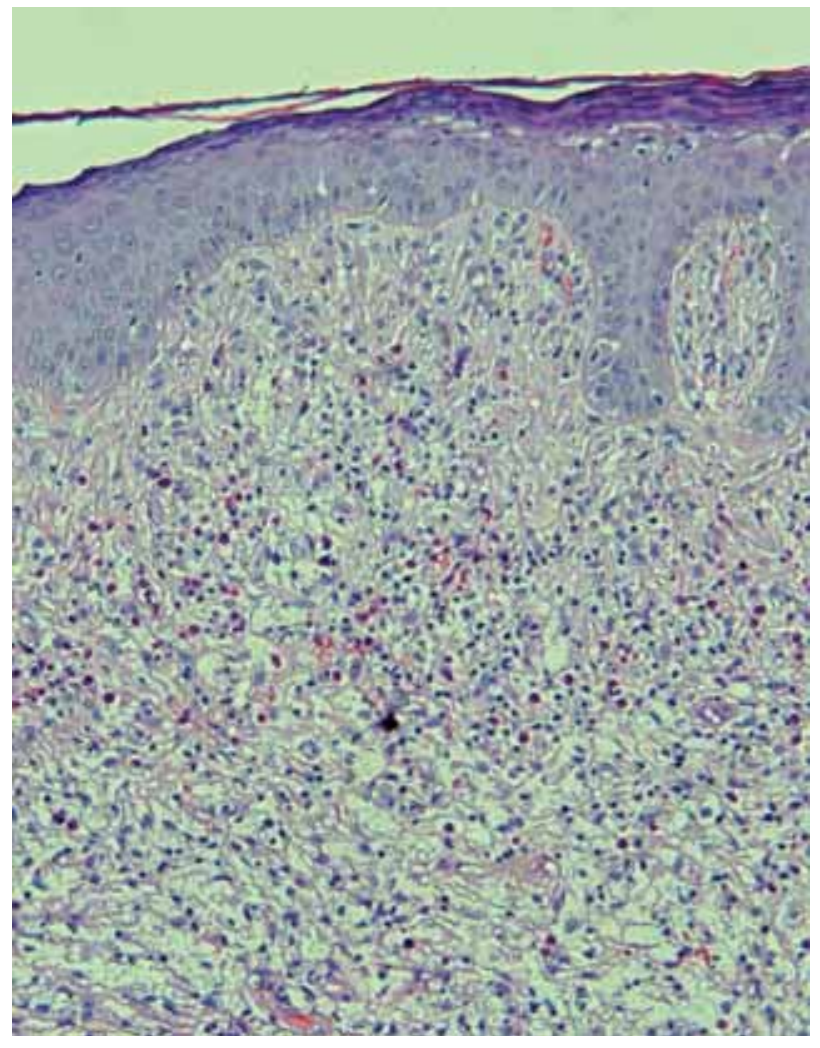

FIGURA 1

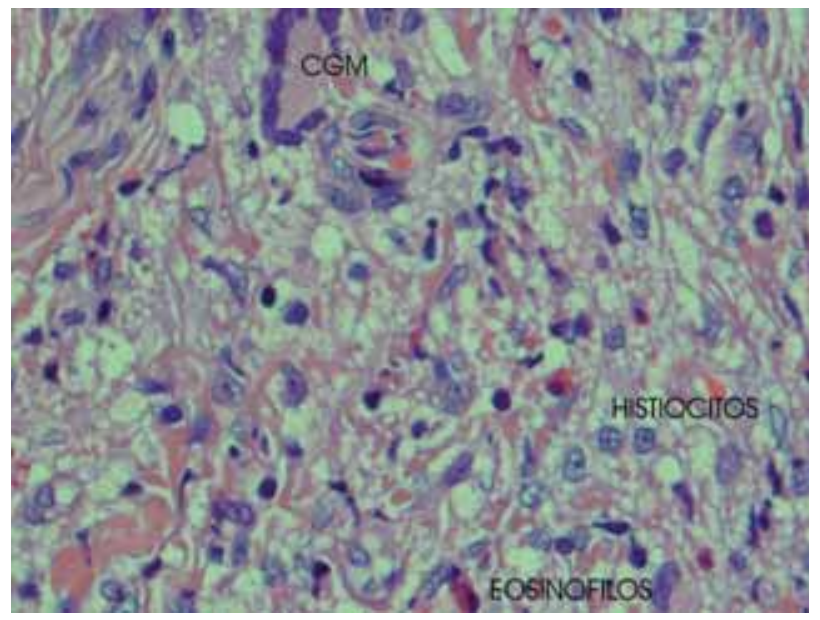

FIGURA 2

Se realizó estudio con técnicas de inmunohistoquímica cuyos resultados fueron: CD68: positivo (Fig. 3); S-100, Ki-67, Cdla y EMA: negativos. El diagnóstico hsitológico fue de XGJ.

El paciente no presenta lesiones en otra localización y no ha habido recidiva a los 6 meses del diagnóstico inicial.

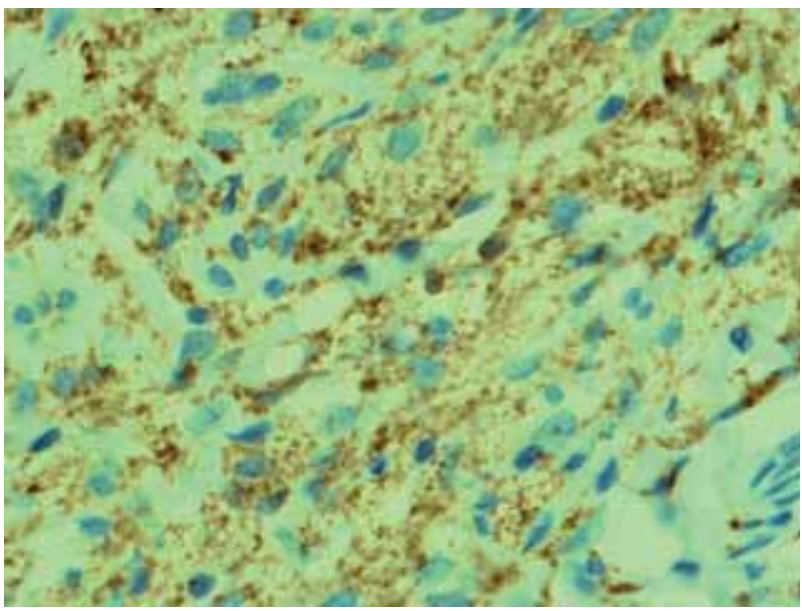

FIGURA 3

\section{DISCUSIÓN}

El xantogranuloma juvenil (XGJ) es una lesión benigna infrecuente descrita por primera vez por Adamson $^{3}$ en 1905 como nevusxantoendotelioma. Aunque puede aparecer a cualquier edad, son las dos primeras décadas de la vida (70\% de los casos) donde existe la mayor incidencia y más de la mitad de estos casos aparecen antes del primer año de vida, con un claro predominio en varones ${ }^{2}$. La piel es la localización más frecuente, pero hay casos descritos con afectación extracutánea como ojos, cavidad oral, sistema nervioso central, pulmón, hígado, bazo y partes blandas ${ }^{1,4-8}$. Macroscópicamente es una entidad bien conocida por pediatras y dermatólogos, pero no tanto por los urólogos, ya que es excepcional su localización a este nivel.

Según la literatura revisada tan sólo hay cinco casos descritos en el testículo ${ }^{8-12}$, dos en el escroto ${ }^{13-14}$ y uno en el pene ${ }^{2}$. En ninguno de estos casos se realizó el diagnóstico antes de la extirpación y en alguno de ellos se pensó en una neoplasia malig$\mathrm{na}^{2,10}$. Nuestro caso clínicamente fue diagnosticado de quiste de inclusión epidérmica y el motivo de la extirpación fue la sintomatología clínica de la lesión.

Los pacientes con XGJ no suelen presentar alteraciones en el metabolismo de los lípidos, pero se han descrito casos asociados a urticaria pigmentosa, diabetes, neurofibromatosis, leucemias e infección por citomegalovirus ${ }^{15}$.

Histológicamente en las fases iniciales de la enfermedad se observan abundantes histiocitos con citoplasmas eosinófilos mezclados con un ligero infiltrado inflamatorio linfocitario y eosinofílico. En 
los casos más evolucionados las células histiocitarias se acompañan de fibroblastos y células gigantes tipo Touton y el infiltrado inflamatorio acompañante suele ser menos significativo. En nuestro caso apreciamos las características histológicas típicas de una lesión en fase inicial.

Histológicamente es importante hacer el diagnóstico diferencial con otras entidades benignas como el reticulohistiocitoma y malignas como la histiocitosis X. En el reticulohistiocitoma no suele haber células gigantes multinucleadas tipo Touton y el infiltrado inflamatorio es muy poco significativo. Las técnicas de inmunohistoquímica no ayudan a establecer el diagnóstico diferencial ya que en ambos casos hay positividad para el CD68, y la S100 suele ser negativa en el XGJ y puede ser positiva o negativa en el reticulohistiocitoma. Con respecto a la histiocitosis X la atípia, mitosis y epidermotropismo son hallazgos histológicos típicos de esta lesión y no se suelen observar en el XGJ. Además en este caso las técnicas de inmunohistoquímica suelen ser concluyentes ya que en la histiocitosis X las células son positivas para S-100 y Cdla que son negativos en el XGJ.

En resumen, aportamos el segundo caso de XGJ de pene en un adulto joven y describimos las características clínicas, histológicas y diagnósticos diferenciales de esta entidad.

\section{REFERENCIAS}

1. Fang-Ying K, Hock-Liew E, Shih-Hao C, Hsuan-Ying $\mathrm{H}$. Intramuscular juvenile xanthogranuloma in an adult. A case report with immunohistochemical study. Arch Pathol Lab Med. 2005;129(2):e31-34.

2. Hautmann RE, Bachor R. Juvenile xanthogranuloma of the penis. J Urol. 1993;150(2):456-457.
3. Adamson HG. A case of congenital xanthoma multiplex. $\mathrm{Br} \mathrm{J}$ Dermatol 1905; 17:222.

4. Margulis A, Mein-Aldana H, Bauer BS. Juvenile xanthogranuoma invading the muscles in the head and neck: clinicopathological case report. Ann Plast Surg. 2003;50(4):425-428.

5. Lesniak MS, Viglione MP, Weingart J. Multicentric parenchymal xanthogranuloma in a child: case report and review of the literature. Neurosurgery. 2002;51(6):1493-1498.

6. Freyer DR, Kennedy R, Bostrom BC, Kohut G, Dehner LP. Juvenile xanthogranuloma forms of systemic disease and their cinical impllications. J Pediatr. 1996;129(2):223-227.

7. Hagmann C, El-Bahrawy M, Stamp G, Abel RM. Juvenile xanthogranuloma: a case report of a preterm baby. Journal of Pediatric Surgery. 2006;41(3):573-575.

8. Nishimura T, Akimoto H, Kawai H, Ohba S, Ohto S. Peritesticular xanthogranuloma. Urology. 1981;18(2):189-190.

9. Wronecki K, Sosnik H, Iwanczak F. Testicular xanthogranuloma juvenile. Pol Tyg Lek. 1984;39(2):51-53.

10. Townell NH, Gledhill A, Robinson T, Hopewell P. Juvenile xanthogranuloma of the testis. J Urol. 1985;133(6):1054-1055.

11. Hewig EB, Hackney VC. Juvenie xanthogranuloma (nevoxantho-endothelioma). Amer J Pathol. 1954;30:625.

12. Nödl F. Systematized large-node nevoxanthoendothelioma. Arch Klin Exp Dermatol. 1959;208(6):601-615.

13. Goulding FJ, Traylor RA. Juvenile xanthogranuloma of the scrotum. J Urol. 1983;129(4):841-842.

14. Nomland R. Nevoxanthro-endothelioma; a benign xanthromatous disease of infants and children. J Invest dermatol. 1954; 22(3):207-215.

15. Cohen BA, Hood A. Xanthogranuloma: report on clinical and histologic findings in 64 patients. Ped Dermatol. 1989;6(4): 262-266.

16. Miettinen M, Fetsch JF. Reticulohistiocytoma (solitary epithelioid histiocytoma). A clinicopathologic and immunohistochemical study of 44 cases. Am J Surg Pathol. 2006;30(4):521528.

Correspondencia autor: Dra. P. San Miguel Fraile

Servicio de Urología. Hospital Povisa

Salamanca, 5 - 36211 Vigo (Pontevedra)

Tel.: 986413144

E-mail autor: psanmiguel@povisa.es

Información artículo: Nota Clínica

Trabajo recibido: enero 2007

Trabajo aceptado: febrero 2007 